\title{
DIRECT NUMERICAL SIMULATIONS OF FLUID FLOW, HEAT TRANSFER AND PHASE CHANGES
}

\author{
Damir Juric \\ MS-B216 \\ Los Alamos National Laboratory \\ Los Alamos, NM 87544 \\ (505) 667-2409
}

\author{
Grétar Tryggvason and Jaehoon Han \\ Department of Mechanical Engineering \\ The University of Michigan \\ Ann Arbor, MI 48109-2121
}

(313) 763-1049

\begin{abstract}
$\underline{\text { Abstract }}$
Direct numerical simulations of fluid flow, heat transfer, and phase changes are presented. The simulations are made possible by a recently developed finite difference/front tracking method based on the "one-field" formulation of the governing equations where a single set of conservation equations is written for all the phases involved. The conservation equations are solved on a fixed rectangular grid, but the phase boundaries are kept sharp by tracking them explicitly by a moving grid of lower dimension. The method is discussed and applications to boiling heat transfer and the solidification of drops colliding with a wall are shown.
\end{abstract}

\section{INTRODUCTION}

In the processing of most engineering materials, the material flows as liquid at some point in the process. Similarly, energy generation in modern societies depends heavily on the combustion of liquid fuels and heat transfer by boiling. However, as important as it is to understand the liquid flow, the complete process almost always involves a phase change where the liquid either solidifies or evaporates. During the last quarter century, enormous progress has been made in predicting fluid flow computationally and most recently the problem of dealing with free surfaces and liquid interfaces has been brought under control. The next challenge is to predict the rest of the process. In most cases, the phase change is critical for the eventual outcome of the operation. The rate of heat transfer in boiling and the combustion rate of sprays depend on how the liquid evaporates. In many manufacturing processes phase change can be critical in determining the final shape and material properties of the final product. Ensuring consistent and desirable material properties of manufactured components is of considerable importance, and to do this several aspects of the solidification process must be controlled. Although the mathematical principles describing both solidification and evaporation are reasonably well understood, their use for predicting the full process is still limited. In space, the presence of a free surface often leads to behavior that is very different from what one would expect based on ground based experiments. Therefore, numerical predictions are essential for the development of novel systems for manufacturing and energy management.

In this paper we will describe our work on two aspects of phase changes in the presence of fluid flow. First we discuss computations of vapor formation due to heating and show an example of boiling. In these simulations the full continuum equations are solved, and the full effect of the phase transition at the interface is included. Second, we present preliminary results for the solidification of drops colliding with a cold wall. Here, the effect of the fluid flow is fully accounted for, but a simplified solidification model, ignoring some of the details of the solidification front is used. Both problems are of considerable current interest. Simulations of boiling can be found in Son and Dhir (1996) and preliminary results for drop solidification can be found in Holt and Tong (1995).

\section{DESCRIPTION OF NUMERICAL METHOD}

We use a unified approach to deal with fluid flow, heat transfer, and phase change. A single set of equations governing the conservation of mass, momentum, and energy are written for all the phases involved and the phase boundary is treated as an imbedded interface by adding the appropriate source terms to the conservation laws. These source terms are in the form of delta functions localized at the interface and are selected in such a way to satisfy the correct matching conditions at the phase boundary. The resulting "one-field" Navier-Stokes equations are (Unverdi and Tryggvason 1992):

$$
\frac{\partial \rho \bar{u}}{\partial t}+\nabla \cdot \rho \bar{u} \bar{u}=-\nabla p+\rho \bar{g}+\nabla \cdot \mu\left(\nabla \bar{u}+\nabla \bar{u}^{T}\right)+\int_{F} \bar{F}_{s} \delta\left(\bar{x}-\bar{x}_{f}\right) d a
$$


Notice that $\rho$ and $\mu$ are, in general, discontinuous. The surface force, $\bar{F}_{s}$ act only on the interface between the different fluids and appears in the current formulation multiplied by a three-dimensional delta function, $\delta$. The integral is over the entire front. It is important to note that this equation contains no approximations beyond those in the usual Navier-Stokes equations. In particular, it contains implicitly the proper stress conditions for the fluid interface. The momentum equation is supplemented by an equation of mass conservation, which for incompressible flows is simply:

$$
\nabla \cdot \bar{u}=0 \text {. }
$$

Since the density and the viscosity are different for the different fluids, it is necessary to track the evolution of these fields by solving the equations of state. Here, however, we assume that the density and viscosity are constant within each phase, so once the interface position is known, these variables can be set. The momentum equation is discretized on a regular staggered grid using second order, centered finite differences for the spatial derivatives and a second order time integration scheme. The continuity equation, (2), when combined with the momentum equation, (1), results in a pressure equation that is not separable (as for homogeneous flow) and is solved by a multigrid package (MUDPACK from NCAR). To advect the material properties and to evaluate the surface tension term in the momentum equation, we track the interface between the different phases explicitly by using a moving grid of one dimension lower than we use for the conservation equations. This grid is usually referred to as a front. The one-field formulation used here is common to other techniques for multifluid flows such as the VOF (Volume of Fluid) and level set methods. In these methods, however, the phase boundary is not tracked explicitly, but reconstructed from a marker function. Explicitly tracking the interface avoids the difficulty of advecting such marker function and allows accurate evaluation of surface forces.

Since the boundary between the fluids (the front) usually undergoes considerable deformation during the phase change, it is necessary to modify the surface mesh dynamically during the course of the computations. The surface mesh is an unstructured grid consisting of points that are linked by elements and both the points and the elements are arranged in a linked list so it is relatively easy to change the structure of the front, including adding and deleting points and elements. Topological changes, such as when bubbles coalesce or drops break in two can also be implemented. This is usually considered a major difficulty in implementing methods that explicitly track the front, but we have shown that with the proper data structure these tasks become relatively straight forward. Although topology changes are easily implemented from a programming point of view, the physics is far from trivial. In reality, drops bounce off each other if the time when the drops are close is shorter than the time it takes to drain a film separating the drops. Usually the film becomes very thin before it breaks, and excessive grid refinement would be required to resolve the draining fully. At the moment, we are dealing with this issue by simply changing the topology of the front at a prescribed time. However, considerable analytical work has been done on film draining and rupturing and we are currently exploring the possibility of combining such a model with our simulations.

The method has been implemented for two- and three-dimensional flows on regular grids and for axisymmetric geometries using stretched grids to allow local grid refinement. It has been applied to a number of multifluid problems and tested and validated in a number of ways, not only to check the implementation, but also to assess its accuracy. Those tests include comparisons with analytical solutions for simple problems, other numerical computations, and experiments. The actual resolution requirement varies with the parameters of the problem. High Reynolds numbers, for example, generally require finer resolution than lower ones, as in other numerical calculations. We have also found that for problems where the surface tension varies, such as for contaminated bubbles and drops moving due to temperature dependent surface tension we generally require finer resolution than for flows where the surface tension is constant. However, in all cases the methods converges rapidly under grid refinement, and in those cases where we have other solutions we have found excellent agreement, even for modest resolutions. Examples of these validations are contained in Esmaeeli and Tryggvason (1996), Yu, Ceccio and Tryggvason (1995), Juric and Tryggvason (1995, 1996a and 1996b), Nobari, Jan and Tryggvason (1996), and Nobari and Tryggvason (1996) where our work on other physical systems, such as bubbly flows, are also discussed.

The approach taken for the fluid flow, works also for heat flow and phase changes. In Juric and Tryggvason (1996) we developed a method to simulate phase changes in a pure material in the absence of any fluid motion. With these assumptions we have only to solve one heat conduction equation:

$$
\frac{\partial \rho c T}{\partial t}+\nabla \cdot \rho c T \bar{U}=\nabla \cdot k \nabla T-\int_{F} \dot{q} \delta\left(\bar{x}-\bar{x}_{F}\right) d a
$$

where $\dot{q}$ is adjusted in such a way that the temperature of the interface is given by the Gibbs-Tompson conditions 


$$
T_{F}=T_{M}\left(1-\frac{\sigma \kappa}{L}\right)
$$

and we have included the fluid flow. Additional terms can be added to the right hand side to account for variability in surface tension and other microscopic effects. We have compared the method with exact solutions for stable solidification and found excellent agreement, even with relatively coarse resolution. The same approach can also be used for the solidification of binary alloys where the solidification temperature depends on the composition of the melt. In this case we also need to solve an equation for the solute concentration (Juric and Tryggvason 1996a and 1996b and Juric 1996).

To add a volume source at the interface to account for local expansion, we work directly with the mass conservation equation

$$
\frac{\partial \rho}{\partial t}+\nabla \cdot \rho \bar{u}=0 .
$$

Away from the interface this reduces to the usual incompressibility condition, but at the interface the local rate of change of density can be written as

$$
\frac{\partial \rho}{\partial t}=\int_{f} \Delta \rho \bar{U} \cdot \bar{n} d a,
$$

which can be combined with the mass conservation equation and the momentum equation to produce a Poisson equation for the pressure. The pressure equation has to be solved in an iterative way along with the advection equation for the momentum. The process is described by Juric (1996).

\section{RESULTS}

Figure 1 shows an example of our work on boiling. Here, a liquid layer is located atop a hot wall and initially a thin film of vapor separates the liquid from the wall. The calculation is done in a 1 by 3 box with grid resolution of 50 by 150 . Other parameters are: $\rho_{l} / \rho_{v}=10, \mu_{l} / \mu_{v}=10, k_{l} / k_{v}=10, c_{l} / c_{v}=1, \operatorname{Pr}=1$, and $\operatorname{Mo}=1$. The liquid/vapor interface next to the wall is unstable and the liquid falls toward the wall as the vapor rises. Phase change, however, leads to evaporation of the liquid, preventing the liquid from colliding with wall and providing vapor to form a bubble that rises upward. The heat transfer during the formation of the bubbles compares relatively well with experimental observations, although the two-dimensionality of the simulation generally leads to smaller values. Other simulations can be found in Juric (1996).

In Juric and Tryggvason (1996) we describe an extension of the method to the solidification of pure materials and use it to examine two-dimensional dentritic growth. However. often, the range of scales between the size of the microstructures and the dimensions of the system we need to predict are very large and resolving the formation of every microstructure is completely impractical. In those cases it is necessary to make some assumptions about what happens at the small scale level in order to be able to resolve the large scale features of the system. How the collective behavior of small scale features is manifested at larger scales is, of course, a central problem in the modeling of physical system and is far from a solved problem. In fluid/solidification simulations some success has been achieved by allowing for a "mushy zone" at the boundary between a fluid and a solid which represents a partially solidified region consisting of fluid and microstructurs such as dendrites. We have done a number of simulations of drops colliding with cold walls with an even simpler model where we simply assume that a melt solidifies if its temperature falls below the solidification temperature, which is taken here to be the average of the wall temperature and the initial temperature of the drop. Although simple, this model captures at least some aspects of the interaction of fluid flow and solidification. Figure 2 shows the collision and solidification of a drop with a "tower" formed by two drops that have already solidified at the wall. The drop Weber number is low (We=3.05) so the drops do not deform much and the drop thermal conductivity is high $(\operatorname{Pr}=1)$ so the drops solidify rapidly. The solidification temperature is set equal to the average of the initial temperature of the drop and the wall temperature. In these simulations we solve for the motion of both the fluid in the drop as well as the ambient fluid and Figure 2 shows the temperature in both the drop and the fluid as well as the streamlines. Since the drop looses heat to the ambient fluid, a thermal wake is clearly visible. We note that unlike some other work on droplet solidification (where the interest is in high speed collisions where the drops form a thin splat) our interest in this problem is motivated by recent work on micro manufacturing by careful deposition of individual drops to build up three dimensional structures (Gao and Sonin 1994). In those cases, droplet deformation is sometimes small and it is important to account accurately for finite Weber number effects. 

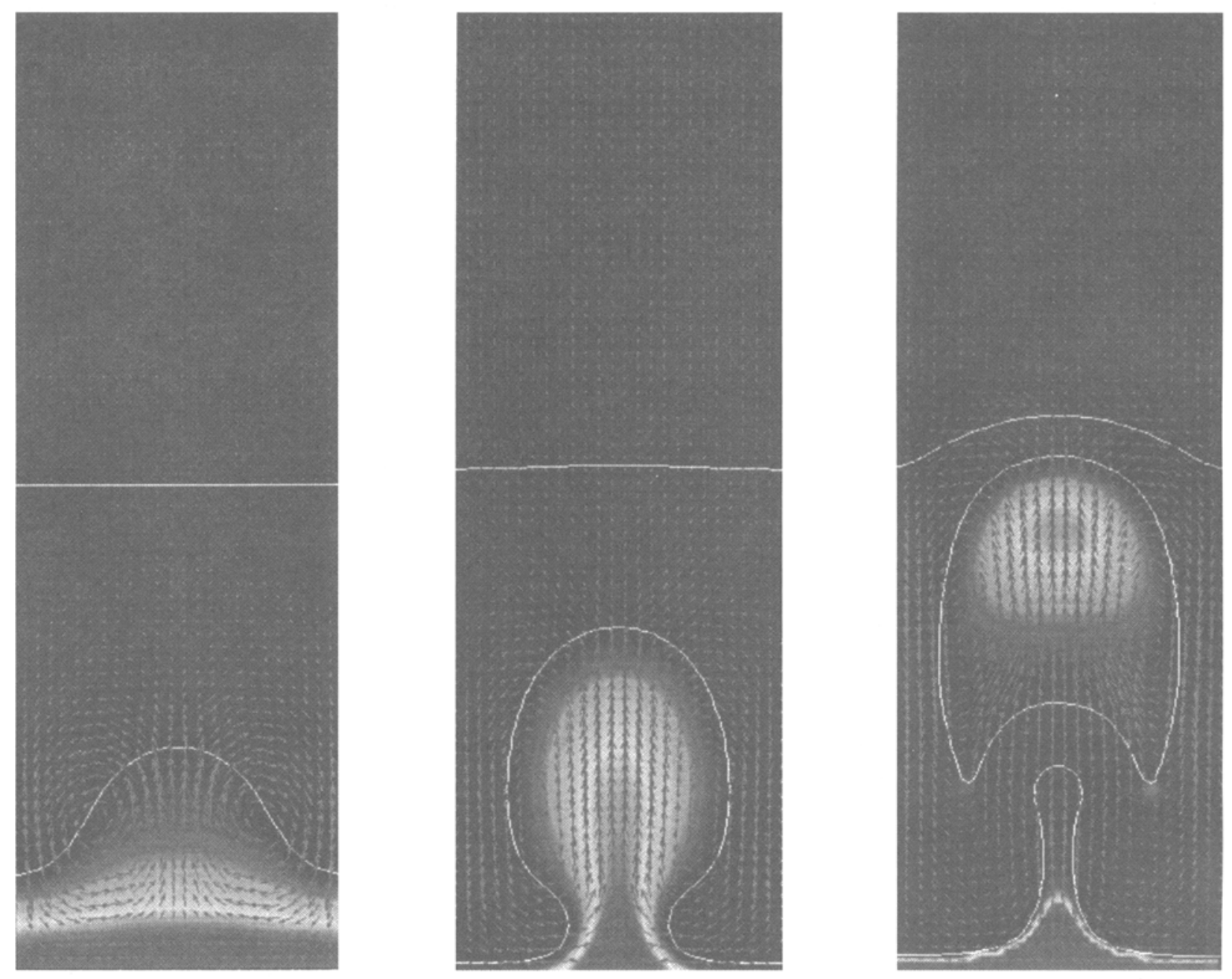

FIGURE 1. Film Boiling Simulation at Three Different Times. The Phase Boundary, the Velocity, and the Temperature Field (in Gray Scale) are Shown.

\section{CONCLUSIONS}

Computational methods for multifluid flows, where two different fluids move together have been developed to the point where such simulations are relatively routine. In order for such simulations to be useful for real engineering applications it is essential to extend the methodology to phase changes. This paper describes two examples of our current work on such problems. Although we have only examined two-dimensional and axisymmetric phase change problems so far, the fluid code has been applied to a number of three-dimensional problems. Therefore, we do not anticipate any major difficulty with the extension to three-dimensions, except for the increased computational requirement.

\section{Acknowledgment}

Our work on direct numerical simulations of multi-fluid flow and solidification has been supported by NASA grant NAG3-1317, NCC3-355, and NASA Graduate Student Fellowship NGT-51070, and NSF grants CTS-913214 and CTS-9503208. The computations have been done at the San Diego Supercomputer Center, NASA Lewis, and the Center for Parallel Computing at the University of Michigan. 

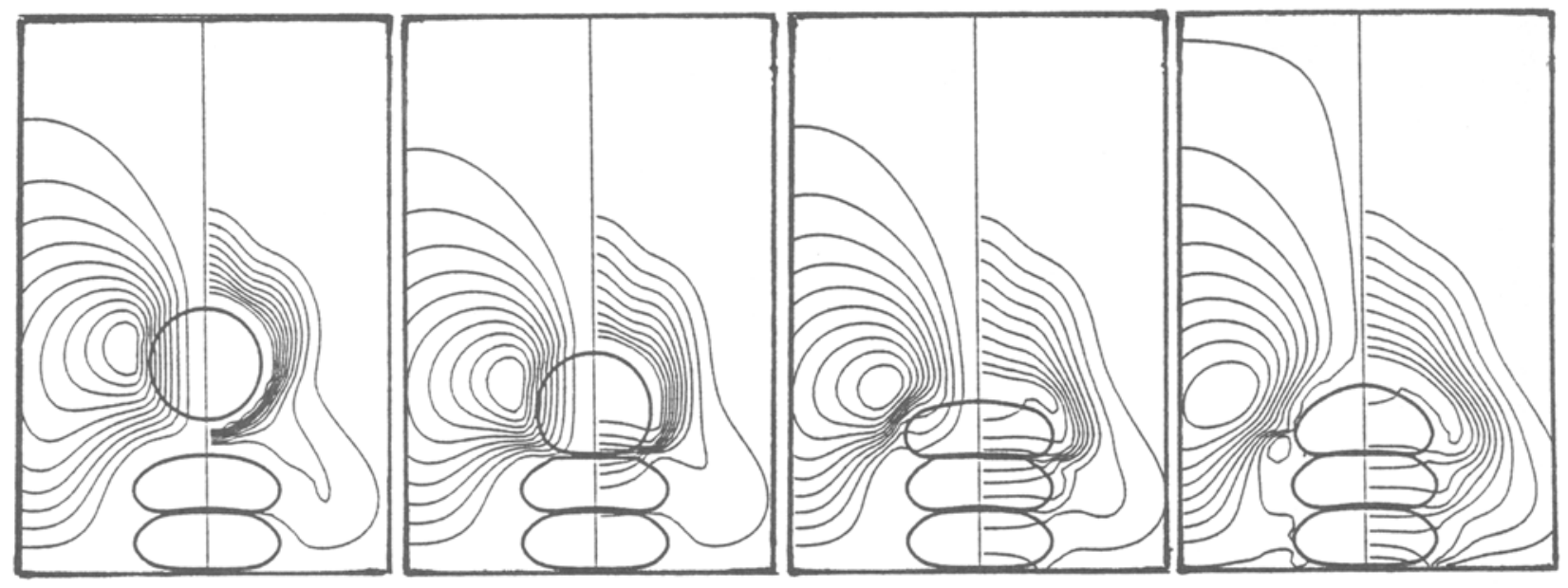

FIGURE 2. Collision of Several Drops with a Cold Wall and the Subsiquent Solidification at Four Times. Streamlines are Shown on the Left, Temperature on the Right.

\section{References}

Esmaeeli, A. and G. Tryggvason (1996) "An Inverse Energy Cascade in Two-Dimensional, Low Reynolds Number Bubbly Flows," J. Fluid Mech. 314:315-330.

Esmaeeli, A. and G. Tryggvason (1996) "Direct Numerical Simulations of Bubbly Flows. Part I-Low Reynolds Number Arrays," Submitted to J. Fluid Mech.

Gao, F. and A. Sonin (1994) "Precise Deposition of Molten Microdrops: the Physics of Digital Microfabrication," Proc. R. Soc. Lond. A 444:533-554.

Holt, B. and A. Tong (1995) "The Normal Incident Impact and Solidification Phenomena of a Liquid Droplet onto a Rigid Substrate," In Proc. ASME Fluid Engineering Division, 215-222.

Jan, Y.-J. and G. Tryggvason (1994) "Computational Studies of Contaminated Bubbles," Submitted to Phys. of Fluids

Juric, D. and G. Tryggvason (1996) "A Front Tracking Method for Dentritic Solidification," J. of Comput. Phys. 123:127-148.

Juric, D. and G. Tryggvason (1996) "Direct Numerical Simulations of Flows with Phase Change," AIAA 96-0857 In Proc. AIAA Aerospace Sciences Meeting.

Juric, D. and G. Tryggvason (1995) "Full Simulations of Flows with Phase Change," AIAA 95-0700. Proc. AIAA Aerospace Sciences Meeting.

Juric, D. (1996) "Computations of Phase Change," Ph. D. Dissertation, The University of Michigan,

Kobayashi, R. (1993) "Modeling and Numerical Simulations of Dentritic Crystal Growth," Physica D 63, 410.

Murray, B. T., A.A. Wheeler, and M.E. Glicksman (1995) "Simulations of Experimentally Observed Dendritic Growth Behavior Using a Phase-Field Model," J. Cryst. Growth 154:386-400.

Nobari, M. R. and G. Tryggvason (1996) "Numerical Simulations of Three-Dimensional Drop Collisions," AIAA Journal 34:750-755. 
Nobari, M. R., Y.-J. Jan and G. Tryggvason (1996) "Head-on Collision of Drops--A Numerical Investigation," Phys. of Fluids $8: 29-42$.

Son, G. and V.K. Dhir (1996). "Two-Dimensional Numerical Simulations of Saturated Film Boiling on a Horizontal Surface," In L.S. Fletcher and T. Aihara (editors), Proc. ASME/JSME Thermal Engineering Joint Conference, Vol. 2. ASME, 257-264.

Unverdi, S. O. and G. Tryggvason (1992) "A Front Tracking Method for Viscous Incompressible Flows," $J$. Comput Phys, 100:25-37.

Yu, P.-Y., S. L. Ceccio, and G. Tryggvason. (1995) "The Collapse of a Cavitation Bubble in Shear Flow-A Numerical Study," Phys. of Fluids 7:2608-2616

\begin{tabular}{lll}
\hline $\bar{u}:$ & Velocity & $\rho:$ Density \\
$\mathrm{p}$ & Pressure & $\mu:$ Viscosity \\
$\mathrm{g}:$ & Gravity acceleration & $\sigma: \quad$ Surface tension coefficient \\
$\mathrm{d}:$ & Drop diameter & $\mathrm{We}=\rho_{d} d U^{2} / \sigma$ Weber number \\
$\mathrm{T}:$ & Temperature & $\mathrm{Re}=\rho d U / \mu \quad$ Reynolds number \\
$\mathrm{T}$ & Temperature at front & $\mathrm{Mo}=\mu_{l}^{4} g / \sigma^{3} \rho_{l}$ Morton number \\
$\mathrm{T}$ & Melting Temperature & $\mathrm{Pr}=\mu_{l} c_{l} / k_{l} \quad$ Prandtl number \\
$\mathrm{q}:$ & Heat source/sink & \multicolumn{2}{l}{} \\
$\mathrm{n}:$ & Normal vector & $\mathrm{Subscript:}$ \\
$\mathrm{U}:$ & Velocity of front & $v:$ vapor \\
$k:$ & Conductivity & $l:$ liquid \\
$c:$ & Heat capacity & $\mathrm{F}:$ Front \\
$\mathrm{L}:$ & Volumetric latent heat &
\end{tabular}

